\title{
The effects of long-term administration of tadalafil on STZ-induced diabetic rats with erectile dysfunction via a local antioxidative mechanism
}

\author{
Yun Chen ${ }^{1}$, Xiao-Xin Li ${ }^{1}$, Hao-Cheng Lin ${ }^{1}$, Xue-Feng Qiu ${ }^{1}$, Jing Gao ${ }^{2}$, Yu-Tian Dai ${ }^{1}$ and Run Wang ${ }^{3}$
}

Type 5 phosphodiesterase inhibitors (PDE5Is) are well known being effective via the nitric oxide and cyclic guanosine monophosphate (NO-cGMP) pathway and are widely used in the treatment of diabetic erectile dysfunction (ED). However, it is unclear whether other pathways may be involved in the treatment of diabetic ED with PDE5Is. The purpose of this study was to clarify the role of antioxidants in diabetic ED treatment through the long-term administration of PDE5Is. Three groups of Sprague-Dawley rats were utilized: Group N, the normal control; Group D, streptozotocin (STZ)-induced diabetic rats as a control; and Group D+T, STZ-induced diabetic rats who received oral administration of tadalafil for 8 weeks. Erectile function was assessed by intracavernous pressure (ICP) and mean arterial pressure (MAP) during electrical stimulation of the cavernous nerve before euthanasia. The levels of malondialdehyde (MDA), superoxide dismutase (SOD) and mitochondrial membrane potential (MMP) of cavernous tissue were assessed by biochemical analysis. The morphology of mitochondria was observed by electron microscopy. The ICP/MAP ratio was higher in Group D+T than in Group $D(P<0.05)$. The levels of MDA decreased and the activities of SOD increased in Group D+T in comparison with Group D $(P<0.05)$. The mitochondrial membrane potential level of cavernous tissue in diabetic rats was partially recovered by tadalafil treatment for 8 weeks. The morphology changes of mitochondria were also remarkably ameliorated in Group D+T. Collectively, the long-term administration of tadalafil in diabetic rats partially reduced oxidative stress lesions of the penis via a local antioxidative stress pathway. Long-term dosages of tadalafil given once daily beginning soon after the onset of diabetes may aid in preventing rats from developing diabetic ED.

Asian Journal of Andrology (2012) 14, 616-620; doi:10.1038/aja.2012.22; published online 16 April 2012

Keywords: diabetes; erectile dysfunction; mitochondria; oxidative stress; PDE5 inhibitor

\section{INTRODUCTION}

The pathogenesis of diabetic erectile dysfunction (ED) is multifactorial, involving neural, vascular and endocrine factors as well as oxidative stress and endothelial cell apoptosis. ${ }^{1-4}$ The treatment of diabetic ED also requires a multistep approach. Among the treatment phases, controlling the blood glucose is the most important step. ${ }^{5}$ Pharmacological management, the use of a vacuum erectile device, intracavernous injection, intraurethral suppository and penile prosthetic implantation are the primary clinical treatments for diabetic ED. ${ }^{6}$ Unless contraindicated, oral type 5 phosphodiesterase inhibitors (PDE5Is) are the first line therapy for diabetic ED patients. PDE5Is diminish the degradation of cGMP via an $L-A r g-N O-c G M P$ pathway to relax vascular smooth muscle and induce penile erection. However, it is unclear whether other pathways may be involved in the treatment of diabetic ED with PDE5Is.

The pathogenesis of ED is complex. ${ }^{7}$ Among the various mechanisms responsible for disease propagation, hyperglycaemia caused by oxidative stress plays an important role. When the balance between the production of oxidative stress by pro-oxidants and the scavenging by antioxidants of excess reactive oxygen species (ROS) is broken, oxidative stress occurs. ROS, formed during regular oxygen molecule metabolism primarily in the vascular endothelium, include, $\mathrm{H}_{2} \mathrm{O}_{2}$ and $\mathrm{OONO}^{-}$. The production of malondialdehyde (MDA), a toxic molecule produced by ROS, is induced by lipid peroxidation of the cellular membrane. The MDA level could represent the degree of lipid peroxidation and oxidative stress in vivo. Superoxide dismutase (SOD) is an important enzyme that removes the superoxide radicals from the human body. Excess amounts of ROS inactivate SOD and lead to decreased removal of superoxide, which further increases the formation of peroxynitrite and reduces the available NO concentration. ${ }^{8}$

Tadalafil, a PDE5I that is unique due to its long half-life $(17.5 \mathrm{~h})$, is a candidate therapy for the rehabilitation of patients with diabetes mellitus. ${ }^{9}$ The once-daily administration of tadalafil was found to be more effective than the on-demand dosage used previously..$^{9-11}$ Recently, more evidence has indicated that oxidative stress plays an important role in the pathogenesis of diabetic ED. Antioxidative therapy, such as with folic acid and a superoxide dismutase analogue, restores erectile 
function in diabetic rats. ${ }^{8,12-13}$ PDE5Is combined with endothelia antioxidant compounds could increase bioavailable $\mathrm{NO}$ and neutralize ROS. ${ }^{14}$ Although it is unclear whether PDE5Is themselves act as the antioxidation mechanism in diabetic ED treatment, there has been no research conducted to elucidate this question. Therefore, we explored the changes of oxidative stress following tadalafil treatment in type- 1 diabetic rats.

\section{MATERIALS AND METHODS}

\section{Experimental model}

Male Sprague-Dawley rats (200-250 g, $n=36$ ) were obtained from the Shanghai Laboratory Animal Center, CAS (SLACCAS, Shanghai, China). All the rats were kept in a temperature-controlled, air-conditioned conventional animal house with a 12-h light-dark cycle and given free access to food and water. The procedures were performed according to the recommendations of the institutional animal care committee.

All the rats fasted for $18 \mathrm{~h}$, were lightly anaesthetized (ketamine, $40 \mathrm{mg} \mathrm{kg}^{-1}$, intramuscular injection) and were injected intraperitoneally with either freshly prepared streptozotocin (STZ) (Sigma Chemical Co., St. Louis, MO, USA) $\left(60 \mathrm{mg} \mathrm{kg}^{-1}, n=26\right)$ or a vehicle as a control $\left(0.1 \mathrm{~mol} \mathrm{l}^{-1}\right.$ citrate-phosphate buffer, $\left.\mathrm{pH} 4.5, n=10\right)$ according to the literature. ${ }^{14,15}$ Blood glucose levels were monitored $72 \mathrm{~h}$ after STZ or vehicle injection at regular intervals throughout the study. Blood samples were obtained from a tail prick, and the blood glucose concentration was measured using a blood glucose meter (Roche, Basel, Switzerland). Only those STZ-induced diabetic rats with serum glucose levels greater than $16.7 \mathrm{~mol} \mathrm{l}^{-1}$ were used ${ }^{15,16}$ in this study as diabetic rats $(n=22)$.

The rats were divided into three groups: Group $\mathrm{N}(n=10)$, the normal control; Group D $(n=11)$, STZ-induced diabetic rats as a control; and Group D+T ( $n=11)$, STZ-induced diabetic rats that were administered oral doses of tadalafil $\left(6 \mathrm{mg} \mathrm{kg}^{-1} \mathrm{day}^{-1}\right)$ for 8 weeks. The rats in Group N and Group D were orally administered starch as a control.

\section{Intracavernous pressure (ICP) measurement}

Following 8 weeks of the tadalafil treatment and an additional week to allow for a washout period, the carotid artery was cannulated (PE-50 tubing) under urethane anaesthesia $\left(0.9 \mathrm{mg} \mathrm{kg}^{-1}\right)$ for the measurement of the mean systemic arterial pressure (MAP). Consequently, the major pelvic ganglion, cavernous nerves and pelvic organs were exposed. The skin overlying the penis was removed, and the right penile crus was exposed by removing part of the overlying ischiocavernous muscle. A 23-gauge needle connected to a PE-50 tube with heparinized saline $\left(250 \mathrm{IU} \mathrm{ml}^{-1}\right.$ ) was carefully inserted into the crus. The other end of the PE-50 tube was connected to a pressure monitor (RM6042B multichannel signal collection processing system; Chengdu Implement Company, Chengdu, China). Cavernous nerve electrostimulation was performed with a stainless-steel bipolar hook electrode using standard settings $(10 \mathrm{~Hz}$, pulse width $5 \mathrm{~ms}$, duration $60 \mathrm{~s}$ and three different voltages: 2.5, 5.0 and 7.5 V). The MAP and ICP were measured and recorded by a computer.

\section{Sample collection and processing}

The penises of all the rats were removed without the skin, glans and corpora spongiosa by dissection and were divided into three portions. The first segment was immediately fixed in $2.5 \%$ glutaric dialdehyde and used for electron microscopy analysis. The second segment was minced, homogenized and centrifuged at $10000 \mathrm{~g}$ for $15 \mathrm{~min}$ at $4{ }^{\circ} \mathrm{C}$ for subsequent SOD and MDA analysis. The last segment was used for the detection of the mitochondrial membrane potential (MMP).

\section{Measurement of SOD and MDA levels}

All the assay kits were purchased from the Nanjing Jiancheng Bioengineering Institute (Nanjing, China). SOD activity was measured through the inhibition of nitroblue tetrazolium reduction by $\mathrm{O}_{2}$, which was generated by the xanthine/xanthine oxidase system. One SOD activity unit was defined as the enzyme amount causing $50 \%$ inhibition in a $1-\mathrm{ml}$ reaction solution per milligram of tissue protein, and the result was expressed as $U$ per $\mathrm{mg}$ protein.

The MDA concentration of the homogenate was measured using the thiobarbituric acid method. The amount of lipid peroxides was measured by the production of MDA, which, in combination with thiobarbituric acid, forms a pink chromogen compound with an absorbance of $532 \mathrm{~nm}$. The result was expressed as nmol per mg protein.

\section{Assessment of advanced glycation endoproduct (AGE) content} AGE levels were measured by a sandwich AGE-ELISA kit (Cell Biolabs, Inc., San Diego, CA, USA). Briefly, either $100 \mu \mathrm{l}$ of the $10 \mu \mathrm{g} \mathrm{ml}^{-1}$ rat protein samples or prepared BSA standards were added to a 96-well protein binding plate and incubated at $37^{\circ} \mathrm{C}$ for $2 \mathrm{~h}$. The wells were washed three times, and $200 \mu \mathrm{l}$ of assay diluent per well were added. The samples were then incubated for $2 \mathrm{~h}$ at room temperature. After washing an additional three times, $100 \mu \mathrm{l}$ of the diluted anti-AGE antibody were added to all the wells, and the samples were incubated for $1 \mathrm{~h}$ at room temperature. A total of $100 \mu \mathrm{l}$ of the diluted secondary antibody-HRP conjugate was added to all the wells and incubated for $1 \mathrm{~h}$ at room temperature. Furthermore, the strip wells were washed five times. A total of $100 \mu \mathrm{l}$ of substrate solution was added to each well, and the samples were incubated at room temperature for $20 \mathrm{~min}$. The enzyme reaction was halted by adding $100 \mu \mathrm{l}$ of stop solution to each well. The absorbance of each well was read on a microplate reader at a wavelength of $450 \mathrm{~nm}$, and the gain data were read from the standard AGE-BSA curve.

\section{Isolation of penile mitochondria}

The mitochondria were prepared from the rats' penile cavernous tissues according to the method used in our lab. ${ }^{17}$ In brief, the tissues were excised and homogenized in an isolation buffer containing $225 \mathrm{mmol} \mathrm{l}^{-1} \mathrm{D}$-mannitol, $75 \mathrm{mmol} \mathrm{l}^{-1}$ sucrose, $0.05 \mathrm{mmol} \mathrm{l}^{-1}$ EDTA and $10 \mathrm{mmol}^{-1}$ Tris- $\mathrm{HCl}(\mathrm{pH} 7.4)$ at $4{ }^{\circ} \mathrm{C}$. The homogenates were centrifuged at $600 \mathrm{~g}$ for $5 \mathrm{~min}$, and the supernatants were centrifuged at $8800 \mathrm{~g}$ for $10 \mathrm{~min}$. The resultant pellet was washed twice with the same buffer, and the protein concentration was then determined.

\section{Measurement of mitochondrial membrane potential}

The mitochondrial membrane potential $\left(\Delta \Psi_{\mathrm{m}}\right)$ was evaluated by the uptake of the fluorescent dye rhodamine 123 (Rh123), which accumulates electrophoretically into energized mitochondria in response to the negative inner membrane potential. Isolated mitochondria were suspended in an assay buffer $\left(0.5 \mathrm{mg}\right.$ protein $\left.\mathrm{ml}^{-1}\right)$ containing $225 \mathrm{mmol} \mathrm{l}^{-1}$ mannitol, $70 \mathrm{mmol} \mathrm{l}^{-1}$ sucrose and $5 \mathrm{mmol} \mathrm{l}^{-1}$ HEPES ( $N$-2-hydroxyethylpiperazine- $N^{\prime}$-2-ethanesulfonic acid) with a $\mathrm{pH}$ of 7.2. The mitochondrial membrane potential $\left(\Delta \Psi_{\mathrm{m}}\right)$ was assessed spectrophotometrically (Hitachi 850$)$ at $25{ }^{\circ} \mathrm{C}$ with an excitation of $505 \mathrm{~nm}$ and detection at $534 \mathrm{~nm}$ after the addition of $0.3 \mathrm{mmol}^{-1} \mathrm{Rh} 123$. The membrane potential was 
calculated by the relationship $m=-59 \log [\mathrm{Rh} 123]_{\text {in }} /[\mathrm{Rh} 123]_{\text {out }}$, assuming the respective distribution of Rh123 between the mitochondria and the medium.

\section{Electron microscopy}

Penile samples for each rat, six per group, were post-fixed for $1 \mathrm{~h}$ in $1 \%$ osmium tetroxide dissolved in the phosphate buffer, dehydrated in graded ethyl alcohols and embedded in epoxy resin. One-micrometrethick sections were obtained from the tissue blocks using a Leica ultramicrotome equipped with glass knives. The sections were stained with toluidine blue, and coverslips were placed over the samples. From the surface of these trimmed blocks, ultrathin sections ranging from 80 to $90 \mathrm{~nm}$ were obtained with a diamond knife and mounted in singleslot grids that had previously been covered with formvar film. The sections were then double-stained with aqueous solutions of uranium acetate and lead citrate, and observed with a JEM 1010 electron microscope (JEOL, Tokyo, Japan).

\section{Statistical analysis}

Differences among all the groups were analysed by a one-way analysis of variance followed by a paired-samples $t$-test, where a $P<0.05$ was accepted as statistically significant. All the experiments were repeated at least three times. The data were presented as mean \pm s.e.

\section{RESULTS}

\section{STZ-induced diabetic rats}

Among the 26 rats treated with STZ, 22 rats developed hyperglycaemia and hyperemesis and had elevated levels of food and water intake compared to the control group. No rats died during the treatment, and their body weights and serum glucose levels are shown in Table 1. The normal control rats became obese $(440.4 \pm 41.8 \mathrm{~g})$ by the end of the study, while the diabetic rats in Group D and Group D+T lost weight (196.7 \pm 28.9 or $203.1 \pm 32.2 \mathrm{~g}$ ). The serum glucose levels in Group D and Group D+T initially rose to $27.5 \pm 6.6$ and $28.1 \pm 6.7 \mathrm{mmol} \mathrm{l}^{-1}$, respectively, and were maintained at $29.3 \pm 7.5$ and $29.9 \pm 7.4 \mathrm{mmol}^{-1}$, respectively, throughout the remainder of the study.

\section{ICP determination}

Three different voltages $(2.5,5.0$ and $7.5 \mathrm{~V})$ were used to measure the MAP and the ICP. The ratio of the maximal ICP/MAP after the electrostimulation of the rats in Group D was $0.42 \pm 0.04$ at $5.0 \mathrm{~V}$ and $0.41 \pm 0.04$ at $7.5 \mathrm{~V}$, which was lower than the ratio in Group $\mathrm{N}$ $(0.79 \pm 0.02$ at $5.0 \mathrm{~V}$ and $0.79 \pm 0.02$ at $7.5 \mathrm{~V})(P<0.05)$ (Figure 1). The daily tadalafil therapy in the diabetic rats (Group $\mathrm{D}+\mathrm{T})$ preserved the ICP/MAP ratios $(0.63 \pm 0.03$ at $5.0 \mathrm{~V}$ and $0.64 \pm 0.03$ at $7.5 \mathrm{~V})$, which were significantly higher in comparison with those in Group $\mathrm{D}(P<0.05)$.

Table 1 Initial and final blood glucose and body weight of rats (mean \pm s.e.)

\begin{tabular}{|c|c|c|c|c|c|}
\hline \multirow{2}{*}{ Group } & \multirow{2}{*}{$n$} & \multicolumn{2}{|c|}{ Body weight (g) } & \multicolumn{2}{|c|}{ Blood glucose $\left(\mathrm{mmol}^{-1}\right)$} \\
\hline & & Initial & Final & Initial & Final \\
\hline Group N & 10 & $211.4 \pm 11.8$ & $440.4 \pm 41.8$ & $6.5 \pm 0.8$ & $6.6 \pm 0.9$ \\
\hline Group D & 11 & $211.7 \pm 12.7$ & $196.7 \pm 28.9^{a}$ & $27.5 \pm 6.6^{a}$ & $29.3 \pm 7.5^{a}$ \\
\hline Group D+T & 11 & $212.9 \pm 11.6$ & $203.1 \pm 32.2^{a}$ & $28.1 \pm 6.7^{a}$ & $29.9 \pm 7.4^{a}$ \\
\hline
\end{tabular}

a $P<0.05$, compared with Group N.

Group N, the normal control; Group D, streptozotocin (STZ)-induced diabetic rats as a control; and Group D+T, STZ-induced diabetic rats who received oral administration of tadalafil for 8 weeks.

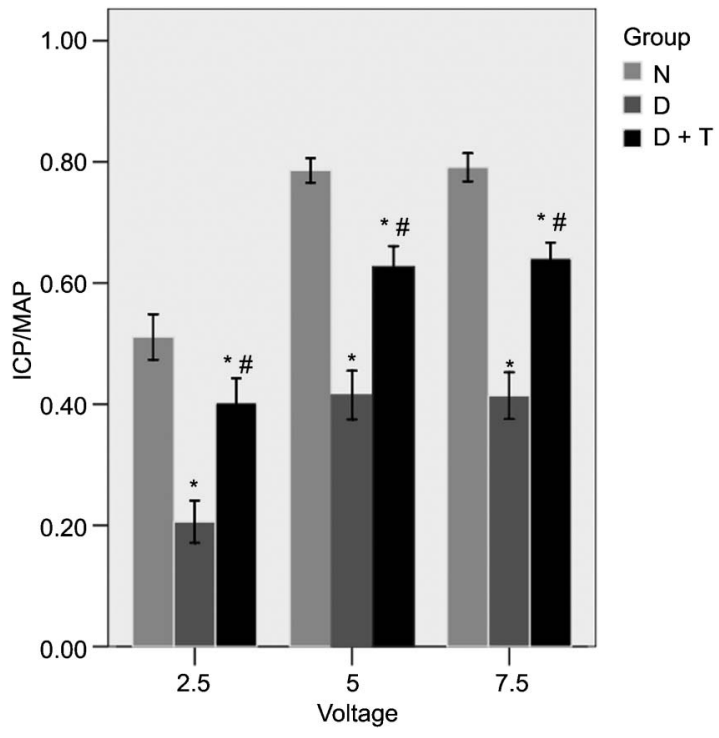

Figure 1 Erectile function evaluation. The results of cavernosometry (10 Hz, pulse width $5 \mathrm{~ms}$, duration $60 \mathrm{~s}$ and voltages of $2.5,5$ and $7.5 \mathrm{~V}$ ). All the data are expressed as mean \pm s.e. ${ }^{*} P<0.05$ vs. Group N. ${ }^{\#} P<0.05$ vs. Group D. The ratio of maximal intracavernous pressure (ICP)/mean arterial pressure (MAP) after electrostimulation of the rats in Group $D+T$ was higher than that in Group $\mathrm{D}(P<0.05)$ but lower than that in Group $N(P<0.05)$. Group $N$, the normal control; Group D, streptozotocin (STZ)-induced diabetic rats as a control; and Group D+T, STZ-induced diabetic rats who received oral administration of tadalafil for 8 weeks.

\section{Levels of SOD, MDA and AGEs in penile homogenates}

SOD activity was measured through the inhibition of nitroblue tetrazolium reduction by $\mathrm{O}_{2}$, which was generated by the xanthine/xanthine oxidase system. The MDA concentration of the penile homogenate was measured using the thiobarbituric acid method. Figure 2a shows that the penile SOD activity in Group $\mathrm{D}+\mathrm{T}$ was $86.8 \pm 4.0$, which was higher than that in Group $\mathrm{D}$ $(73.3 \pm 6.6)(P<0.05)$. The penile MDA level in Group $\mathrm{D}+\mathrm{T}$ was $3.90 \pm 0.41$, which was lower than that in Group D $(6.16 \pm 1.07)$ $(P<0.05)$ (Figure 2b). The sandwich AGE-ELISA kit was used to measure the levels of AGEs in the penile homogenates of each group. The AGE level in the penile homogenates of Group D+T

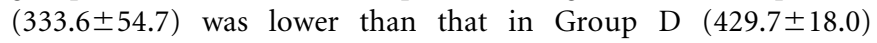
$(P<0.05)$, but it was higher than that in group $\mathrm{N}(203.4 \pm 26.0)$ $(P<0.05)$ (Figure 2c).

\section{MMP}

Under the experimental conditions used in this study, the MMP of the normal control rats was $-186.72 \pm 2.59 \mathrm{mV}$. This value dropped to $-168.76 \pm 2.47 \mathrm{mV}(P<0.05)$ after the rats underwent diabetic induction (Figure 3). Following the long-term administration of tadalafil, the MMP in Group D+T was partially restored $(-172.75 \pm 3.07 \mathrm{mV})$ $(P<0.05)$; however, it still remained abnormal.

The mitochondrial ultrastructure of penile endothelial cells To determine whether diabetes affects the mitochondrial structure in cavernous endothelial cells, transmission electron microscopy was performed on penile endothelial cells from the normal control rats, the diabetic control rats and the diabetic rats treated with tadalafil. Representative photomicrographs are shown in Figure 4. Ultrastructural changes, namely, mitochondrial disintegration, lipid 

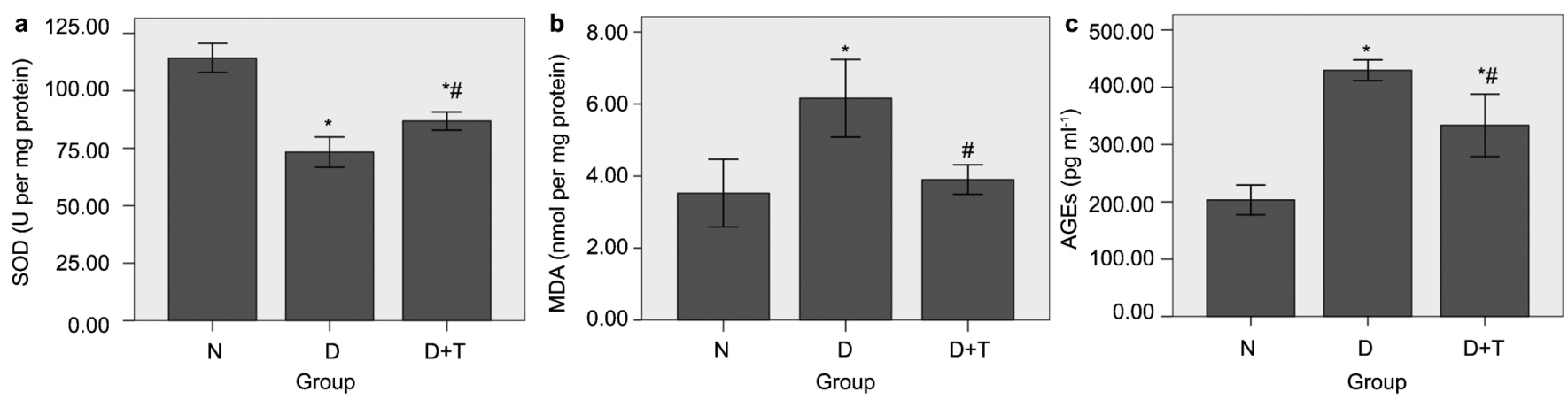

Figure 2 Superoxide dismutase (SOD) activity (a), malondialdehyde (MDA) levels (b) and advanced glycation endoproduct (AGE) levels (c) in penile homogenates. All the data are expressed as mean \pm s.e. $* P<0.05$ vs. Group N. ${ }^{*} P<0.05$ vs. Group D. (a) The penile SOD activity was significantly higher in Group $D+T$ compared to that in Group $D(P<0.05)$. (b)The level of MDA in the penile homogenates of Group $D+T$ was lower than that in Group $D(P<0.05)$. (c) The level of $A G E s$ in the penile homogenates of Group D+T was lowerthan that in Group D $(P<0.05)$ but higher than that in Group N $(P<0.05)$. Group N, the normal control; Group D, streptozotocin (STZ)-induced diabetic rats as a control; and Group D+T, STZ-induced diabetic rats who received oral administration of tadalafil for 8 weeks.

deposition and nuclear condensation, were evident in the penile endothelial cells of STZ-induced diabetic rats when compared with cells from the control group. However, the ultrastructural changes found in the diabetic rats treated with tadalafil were remarkably improved.

\section{DISCUSSION}

PDE5Is are agents that inhibit PDE5, thereby diminishing the degradation of cGMP and improving erectile function. PDE5Is are known to be effective via the NO-cGMP pathway and are widely used in the treatment of diabetic ED. ${ }^{6}$ In our study, the erectile function of Group $\mathrm{D}+\mathrm{T}$ was much better than that of Group D, thereby providing evidence for the efficacy of the once-daily administration of oral tadalafil in the treatment of diabetic ED. However, the tadalafil treatment was not able to normalize the erectile function for a variety of reasons. For example, we used STZ to induce type I diabetes in our rat model for convenience. These diabetic rats were thin and sick after 8 weeks. We also did not control the serum glucose levels of the diabetic rats. The

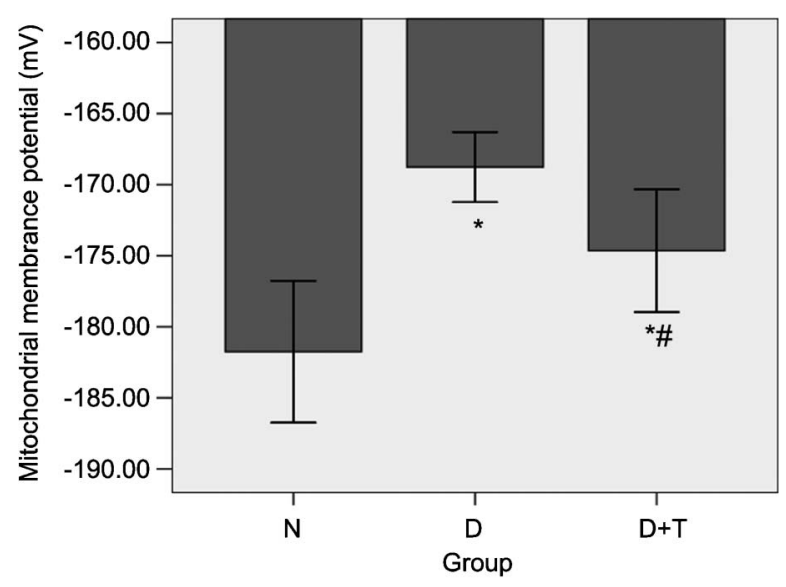

Figure 3 The mitochondrial membrane potential (MMP) levels of cavernous tissue. All the data are expressed as the mean \pm s.e. $* P<0.05$ vs. Group N. ${ }^{\#} P<0.05$ vs. Group D. The daily tadalafil therapy in the diabetic rats partially restored the MMP levels compared with those in the diabetic ED rats. Group N, the normal control; Group D, streptozotocin (STZ)-induced diabetic rats as a control; and Group D+T, STZ-induced diabetic rats who received oral administration of tadalafil for 8 weeks. damage caused by hyperglycaemia continued for 9 weeks during the study. Additionally, the pathogenesis of diabetic ED is multifactorial and complex.

PDE5Is have recently been shown to reduce oxidative stress in other organs. The administration of sildenafil has been shown to reduce oxidative stress and protect the heart against pressure overloadinduced left ventricular hypertrophy and congestive heart failure. ${ }^{18}$ Incubation of endothelial cells from the human pulmonary artery in sildenafil resulted in the attenuation of the oxidant burden and enabled the assessment of the inflammatory and remodelling effects of cigarette smoke. ${ }^{19}$ Pre-treatment with vardenafil has been shown to significantly reduce DNA breakage and nitrosative stress but increase the cGMP score in the aortic wall. ${ }^{20}$ Sildenafil reduces superoxide formation and increases the levels of cGMP, cAMP and glutathione in rabbit-isolated corpus cavernosum and in hypertensive rats. ${ }^{21}$ Chronic treatment with sildenafil restores the normal excretion of urinary biological markers of oxidative stress and cyclooxygenasederived vasoconstrictors. $^{22}$

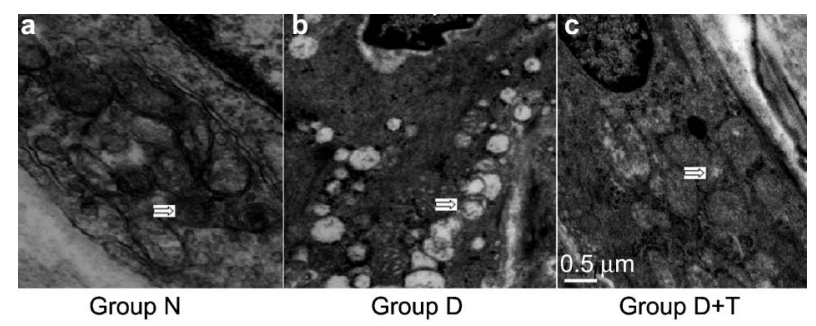

Figure 4 The mitochondrial ultrastructure of penile endothelial cells. The cavernous tissues of each group were subjected to transmission electron microscopy analysis, and the mitochondrial structures were compared. Specimens were prepared for examination under an electron microscope (50 000 × magnification). (a) Representative electron micrograph of mitochondria from the penis of a normal control rat showing bean-shaped structures with numerous transversely orientated cristae enveloped by an intact outer membrane (white arrows in left figure). (b, c) Representative electron micrographs show alterations in the mitochondrial morphology of diabetic cavernous endothelial cells. (b) Ultrastructural changes, namely mitochondrial disintegration, lipid deposition and nuclear condensation (white arrows in middle figure), were evident in the penile endothelial cells of the streptozotocin (STZ)-induced diabetic rats when compared with cells from the control group. (c) Tadalafil treatment was effective in partially restoring the mitochondrial ultrastructural changes (white arrows in right figure). Group N, the normal control; Group D, STZ-induced diabetic rats as a control; and Group D+T, STZ-induced diabetic rats who received oral administration of tadalafil for 8 weeks. 
The antioxidative stress mechanism of PDE5I treatment in diabetic ED is unclear. Some studies have found that PDE5Is combined with an antioxidant agent are more effective in treating diabetic ED compared to the use of PDE5Is alone. Treatment using both $L$-carnitine and sildenafil reduced monocyte oxidative activity and the amount of endothelial dysfunction markers in diabetic patients with ED. ${ }^{23}$ The combination of vitamin $\mathrm{E}$ and sildenafil has been shown that the staining of neuronal nitric oxide synthase, endothelial cell and smooth muscle cell was restored. ${ }^{24}$ The administration of endothelial antioxidant compounds to patients with arterial ED improved the success rate of the sildenafil treatment. ${ }^{25}$

Tadalafil, which has multiple beneficial properties including a long half-life, may be a more effective antioxidative agent than sildenafil for persistent administration. Tadalafil appears to be beneficial in reducing the effects of injury to the spinal cord by increasing the tissue levels of NO and the serum activity of SOD. ${ }^{26}$ In a model of a fetal rat brain with ischemia/reperfusion injury, tadalafil was found to be more effective than sildenafil because it increased SOD and glutathione peroxidase activities. $^{27}$

Our data showed that tadalafil treatment could decrease MDA levels, increase the activity of SOD and remove the AGEs in the cavernous tissues of diabetic rats. We discovered that tadalafil is not only a PDE5 inhibitor, but also an effective antioxidation agent for the penis. This effective pathway for the treatment of diabetic ED through the use of PDE5Is is a novel finding. We used a type I diabetic rat model in this study, and the rats were treated immediately after the induction of diabetes. The tadalafil treatment in this study was found to prevent diabetic ED.

Mitochondria, the power centre of cells, are also the main manufactory agent of ROS. Excessive amounts of ROS induce oxidative stress and impair mitochondrial function. Increased ROS production leads to the formation of an internal cycle where the cells become necrotic or apoptotic. Our data indicate that chronic tadalafil treatment could restore the MMP levels and mitochondrial ultrastructure of the cavernous tissues of diabetic ED rats. The changes in MMP levels and mitochondrial ultrastructure indicated impaired mitochondrial function. The long-term administration of tadalafil in diabetic rats could restore erectile function via a local antioxidative mechanism.

In conclusion, the long-term administration of tadalafil in diabetic rats partially reduced the oxidative stress lesion of the penis via a local antioxidative stress pathway. The long-term administration of once-daily dosages of tadalafil beginning soon after diabetes onset may partially prevent rats from developing diabetic erectile dysfunction. To our knowledge, this is the first report to reveal a new mechanism associated with long-term tadalafil treatment in diabetic ED.

\section{AUTHOR CONTRIBUTIONS}

YC, conception and design, drafting the article, analysis and interpretation of data, revising for intellectual content. XXL, acquisition of data, analysis and interpretation of data. HCL and XFQ, acquisition of data. JG, conception and design, analysis and interpretation of data. YTD and RW, conception and design, revising it for intellectual content, final approval of the completed article.

\section{COMPETING FINANCIAL INTERESTS}

The authors declare no competing financial interests.

\section{ACKNOWLEDGMENTS}

This study was supported by a grant from the National Natural Science Foundation of China (No. 30801143), and by a grant from the overseas scholarship of Jiangsu Province, China (No. 2009K007).

1 Cameron NE, Cotter MA. Erectile dysfunction and diabetes mellitus: mechanistic considerations from studies in experimental models. Curr Diabetes Rev 2007; 3: 149-58. 2 Malavige LS, Levy JC. Erectile dysfunction in diabetes mellitus. J Sex Med 2009; 6: 1232-47.

3 Costa C, Soares R, Castela A, Adães S, Hastert V et al. Increased endothelial apoptotic cell density in human diabetic erectile tissue-comparison with clinical data. J Sex Med 2009; 6: 826-35.

4 Moore CR, Wang R. Pathophysiology and treatment of diabetic erectile dysfunction. Asian J Androl 2006; 8: 675-84.

5 Lu CC, Jiann BP, Sun CC, Lam HC, Chu CH et al. Association of glycemic control with risk of erectile dysfunction in men with type 2 diabetes. J Sex Med2009; 6: 1719-28.

6 Chen Y, Dai Y, Wang R. Treatment strategies for diabetic patients suffering from erectile dysfunction. Expert Opin Pharmacother 2008; 9: 257-66.

7 Nangle MR, Cotter MA, Cameron NE. IkappaB kinase 2 inhibition corrects defective nitrergic erectile mechanisms in diabetic mouse corpus cavernosum. Urology 2006; 68: 214-8.

8 Hirata H, Kawamoto K, Kikuno N, Kawakami T, Kawakami K et al. Restoring erectile function by antioxidant therapy in diabetic rats. J Urol 2009; 182: 2518-25.

9 Bruzziches R, Greco EA, Pili M, Francomano D, Spera G et al. Redefining the role of long-acting phosphodiesterase inhibitor tadalafil in the treatment of diabetic erectile dysfunction. Curr Diabetes Rev 2008; 4: 24-30.

10 Donatucci CF, Wong DG, Giuliano F, Glina S, Dowsett SA et al. Efficacy and safety of tadalafil once daily: considerations for the practical application of a daily dosing option. Curr Med Res Opin 2008; 24: 3383-92.

11 Lundberg GD. Daily tadalafil prevents erectile dysfunction in diabetic men. Medscape J Med 2008; 10: 127.

12 Kawakami T, Urakami S, Hirata H, Tanaka Y, Nakajima K et al. Superoxide dismutase analog (Tempol: 4-hydroxy-2,2,6,6-tetramethylpiperidine 1-oxyl) treatment restores erectile function in diabetes-induced impotence. Int J Impot Res 2009; 21: 348-55.

13 Shukla N, Hotston M, Persad R, Angelini GD, Jeremy JY. The administration of folic acid improves erectile function and reduces intracavernosal oxidative stress in the diabetic rabbit. BJU Int 2009; 103: 98-103.

14 Vicari E, La Vignera S, Condorelli R, Calogero AE. Endothelial antioxidant administration ameliorates the erectile response to PDE5 regardless of the extension of the atherosclerotic process. J Sex Med 2010; 7: 1247-53.

15 Yang R, Yang B, Wen Y, Fang F, Cui S et al. Losartan, an angiotensin type I receptor, restores erectile function by downregulation of cavernous renin-angiotensin system in streptozocin-induced diabetic rats. J Sex Med 2009; 6: 696-707.

16 Chen Y, Yang R, Yao L, Sun Z, Wang R et al. Differential expression of neurotrophins in penises of streptozotocin-induced diabetic rats. J Androl 2007; 28: 306-12.

17 Xu L, Gao J, Wang Y, Yu W, Zhao X et al. Myrica rubra extracts protect the liver from CCI4induced damage. Evid Based Complement Alternat Med 2011; 2011: Article 518302.

18 Lu Z, Xu X, Hu X, Lee S, Traverse JH et al. Oxidative stress regulates left ventricular PDE5 expression in the failing heart. Circulation 2010; 121: 1474-83.

19 Milara J, Juan G, Ortiz JL, Guijarro R, Losada M et al. Cigarette smoke-induced pulmonary endothelial dysfunction is partially suppressed by sildenafil. Eur $\mathrm{J}$ Pharm Sci 2010; 39: 363-72.

20 Korkmaz S, Radovits T, Barnucz E, Neugebauer P, Arif R et al. Dose-dependent effects of a selective phosphodiesterase-5-inhibitor on endothelial dysfunction induced by peroxynitrite in rat aorta. Eur J Pharmacol 2009; 615: 155-62.

21 Shukla N, Rossoni G, Hotston M, Sparatore A, del Soldato P et al. Effect of hydrogen sulphide-donating sildenafil (ACS6) on erectile function and oxidative stress in rabbit isolated corpus cavernosum and in hypertensive rats. BJU Int 2009; 103: 1522-9.

22 Behr-Roussel D, Oudot A, Compagnie S, Gorny D, Le Coz O et al. Impact of a long-term sildenafil treatment on pressor response in conscious rats with insulin resistance and hypertriglyceridemia. Am J Hypertens 2008; 21: 1258-63.

23 Morano S, Mandosi E, Fallarino M, Gatti A, Tiberti C et al. Antioxidant treatment associated with sildenafil reduces monocyte activation and markers of endothelial damage in patients with diabetic erectile dysfunction: a double-blind, placebocontrolled study. Eur Urol 2007; 52: 1768-74.

24 de Young L, Yu D, Bateman RM, Brock GB. Oxidative stress and antioxidant therapy: their impact in diabetes-associated erectile dysfunction. J Androl 2004; 25: 830-36.

25 Vicari E, La Vignera S, Condorelli R, Calogero AE. Endothelial antioxidant administration ameliorates the erectile response to PDE5 regardless of the extension of the atherosclerotic process. J Sex Med 2010; 7: 1247-53.

26 Serarslan Y, Yönden Z, Ozgiray E, Oktar S, Güven EO et al. Protective effects of tadalafil on experimental spinal cord injury in rats. J Clin Neurosci2010; 17: 349-52.

27 Ozdegirmenci O, Kucukozkan T, Akdag E, Topal T, Haberal A et al. Effects of sildenafil and tadalafil on ischemia/reperfusion injury in fetal rat brain. J Matern Fetal Neonatal Med 2011; 24: 317-23. 